\title{
Nocturnal mnemonics: sleep and hippocampal memory processing
}

\author{
Jared M. Saletin ${ }^{1}$ and Matthew P. Walker ${ }^{1,2}$ * \\ 1 Sleep and Neuroimaging Laboratory, Department of Psychology, University of California, Berkeley, CA, USA \\ ${ }^{2}$ Helen Wills Neuroscience Institute, University of California, Berkeley, CA, USA
}

\section{Edited by:}

Sean P. Drummond, University of California San Diego, USA

\section{Reviewed by:}

Mitsuyuki Nakao, Tohoku University, Japan

Michael Czisch, Max Planck Institute of Psychiatry, Germany

${ }^{*}$ Correspondence:

Matthew P. Walker, Department of Psychology, University of California Tolman Hall 3331, Berkeley, CA 94720-1650, USA.

e-mail:mpwalker@berkeley.edu
As critical as waking brain function is to learning and memory, an established literature now describes an equally important yet complementary role for sleep in information processing. This overview examines the specific contribution of sleep to human hippocampal memory processing; both the detriments caused by a lack of sleep, and conversely, the proactive benefits that develop following the presence of sleep. First, a role for sleep before learning is discussed, preparing the hippocampus for initial memory encoding. Second, a role for sleep after learning is considered, modulating the post-encoding consolidation of hippocampal-dependent memory. Third, a model is outlined in which these encoding and consolidation operations are symbiotically accomplished, associated with specific NREM sleep physiological oscillations. As a result, the optimal network outcome is achieved: increasing hippocampal independence and hence overnight consolidation, while restoring next-day sparse hippocampal encoding capacity for renewed learning ability upon awakening. Finally, emerging evidence is considered suggesting that, unlike previous conceptions, sleep does not universally consolidate all information. Instead, and based on explicit as well as saliency cues during initial encoding, sleep executes the discriminatory offline consolidation only of select information. Consequently, sleep promotes the targeted strengthening of some memories while actively forgetting others; a proposal with significant theoretical and clinical ramifications.

\section{Keywords: sleep, hippocampus, memory, encoding, consolidation, forgetting, sleep spindles}

Hello darkness, my old friend

I've come to talk with you again

Because a vision softly creeping

Left its seeds while I was sleeping

And the vision that was planted in my brain

Still remains

Within the sound of silence

-Sound of Silence-

Paul Simon \& Art Garfunkel, February 1964

\section{INTRODUCTION}

Beyond anecdotal, literary and even lyrical acknowledgments, a rapidly expanding corpus of scientific evidence supports a causal role for sleep in memory processing. This review aims to provide a synthesis of recent findings in humans, with a focus on hippocampal-dependent memory. Our goal is to extract consistent themes across domains of memory function that appear to be regulated by sleep, and provide a framework of sleep-dependent hippocampal memory processing within which these findings can be understood. We examine two stages of memory processing (1) a role for sleep before learning in preparing the hippocampus for initial formation or "encoding" of new experiences, and (2) a role for sleep after learning in both non-specific and specific offline consolidation of recently encoded experiences.

\section{SLEEP BEFORE LEARNING FOR MEMORY ENCODING}

Some of the earliest evidence describing the impact of prior sleep loss on subsequent learning of declarative memories was described by Morris et al. (1960), and later by Harrison and Horne (2000), demonstrating impairment in encoding and retention of "temporal memory" (memory for when events occur). In the latter study, significant impairments in memory were evident even in a subgroup that received caffeine to overcome non-specific effects of lower alertness. Interestingly, sleep-deprived subjects displayed significantly worse insight into their memory encoding performance, resulting in lower predictive ability of performance, a function itself that may also rely in part upon the hippocampus (Huijbers et al., 2011).

Pioneering work by Drummond et al. (2000) has examined the neural basis of similar memory impairments using fMRI, investigating the effects of $35 \mathrm{~h}$ of total sleep deprivation on verbal learning. In those who were sleep-deprived, regions of the temporal lobe were significantly less active during learning, relative to a control group that had slept, while the prefrontal cortex actually expressed greater activation. Most interesting, the parietal lobes, which were not activated in the control group during learning, were significantly more active in the deprivation group. Such findings suggest that sleep loss prior to learning (at least following one night) produces bi-directional changes in verbal encoding activity, involving the inability of the temporal lobe regions to engage normally during learning, combined with potential 
compensation attempts by higher cortical regions (Drummond et al., 2001).

The impact of sleep loss on the specific neural dynamics of hippocampal memory encoding have been examined using eventrelated fMRI (Yoo et al., 2007a). In addition to performance impairments under conditions of sleep deprivation, and relative to a control group that slept, a highly significant yet selective deficit was identified in bilateral regions of the posterior hippocampus, known to be critical for learning new episodic information (Figure 1). These findings have since been extended by Van Der Werf et al. (2009), demonstrating that selective slow wave sleep deprivation alone is sufficient to impair hippocampal memory encoding ability. Taken together, this collection of findings indicate that sleep disruption as well as total sleep deprivation prior to learning compromises the function of human hippocampus to effectively commit new human experiences to memory.

The impact of sleep deprivation on memory formation does not appear to be universal, but instead, may differ on the basis of factors such as emotionality. We have examined the encoding of emotionally negative, positive, and neutral words (Walker, unpublished results). Across all stimulus types combined, sleep deprivation imposed a $40 \%$ reduction in the ability to form new memories, relative to sleep rested individuals (Figure 2A). However, when these data were separated into the three affective categories (negative, positive, or neutral), the magnitude of encoding impairment differed (Figure 2B). In those that had slept, both positive and negative stimuli were associated with superior retention levels relative the neutral condition, consonant with the notion that emotion facilitates memory encoding (Cahill and McGaugh, 1998). However, there was severe disruption of encoding and hence later retention for neutral and especially positive emotional memory in the sleep-deprived group. In contrast, a relative resistance of negative emotional memory was observed in the deprivation group. These data suggest that, while the effects of sleep deprivation are

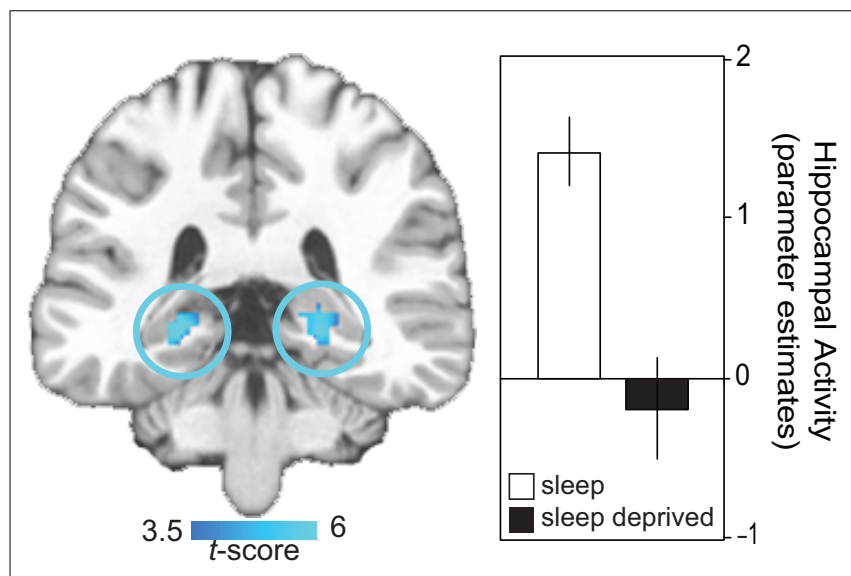

FIGURE 1 | Neural basis of sleep-deprivation induced encoding deficits. Regions of decreased encoding activation in the sleep deprivation group relative to the sleep control group in bilateral posterior hippocampus, together with a histogram of parameter estimates (effect size) of averaged hippocampal activity in each group. Effects are significant at $p<0.001$; $>5$ contiguous voxels. Modified from Yoo et al. (2007a). directionally consistent across memory subcategories, the most profound impact is on the encoding of positive emotional stimuli, and to a lesser degree, emotionally neutral stimuli. In contrast, the encoding of negative memory appears to be more resistant to the effects of one night of total sleep deprivation.

While such evidence describes the detrimental impact of a lack of sleep, recent work has conversely demonstrated the proactive benefit of sleep, and specific sleep physiology, in restoring episodic memory encoding ability (Mander et al., 2011). Hippocampaldependent learning capacity was assessed twice across a $6 \mathrm{~h}$ interval. Following the first learning session at 12:00, half of the subjects remained awake, while the other half obtained a $100 \mathrm{~min}$ nap opportunity. Both groups then performed the second learning session at 18:00. Hippocampal-dependent learning deteriorated across the day in those who remained awake (Figure $3 \mathrm{~A}$ ). In contrast, sleep not only blocked this deterioration in learning capacity, but triggered a numeric enhancement in hippocampal-dependent encoding ability. Within the nap group, the extent of learning restoration (pre- to post-sleep) was positively correlated with both the amount of stage-2 NREM, and specifically the number of fast sleep spindles over the left prefrontal cortex (Figures 3B-D). Moreover, and motivated by the role of the lateral prefrontal cortex and medial temporal lobe structures in episodic memory, EEG source analysis of these left prefrontal spindles was performed, revealing an oscillation of activity throughout the spindle event looping through the left temporal lobe (Figure 3E). Such findings can parsimoniously be accounted for within a hippocampalneocortical framework of memory processing (discussed in the following section), predicting decreased episodic learning capacity with continued waking experience; a potential limitation of sparse hippocampal representational coding (Treves et al., 1996). Consequently, NREM sleep spindles, which co-occur with hippocampal sharp-wave ripples (Siapas and Wilson, 1998; Clemens et al., 2007, 2011; Mölle et al., 2009), are proposed to support a proactive shift from hippocampal- to increasing cortical-dependence of previously encoded representations, thereby restoring post-sleep episodic encoding ability.

Of note, neither the impairments in encoding following deprivation (Yoo et al., 2007a) nor the restoration of encoding following a nap (Mander et al., 2011) appear to be parsimoniously accounted for on the basis of changes in basic alertness or attention. Specifically, response times during encoding and testing in both studies, often used as an indexing of alertness, did not predict learning ability or associated encoding brain activity, and were not different between the respective control groups in either study. This was similarly true of subjective measure of alertness. Moreover, in the nap study (Mander et al., 2011), no differences were observed between the sleep and no sleep groups on a specific alertness control task.

One consideration when interpreting the findings of studies that compare between evening and morning learning and testing phases are the inherent changes in alertness and fatigue at these different times, as well as changes in circadian clock time. In addition to measuring objective and subject alertness and attention as potential confounding factors, a nap-paradigm can also be advantageous in this context (e.g., Mander et al., 2011), allowing for a manipulation of sleep and wakefulness whilst holding 

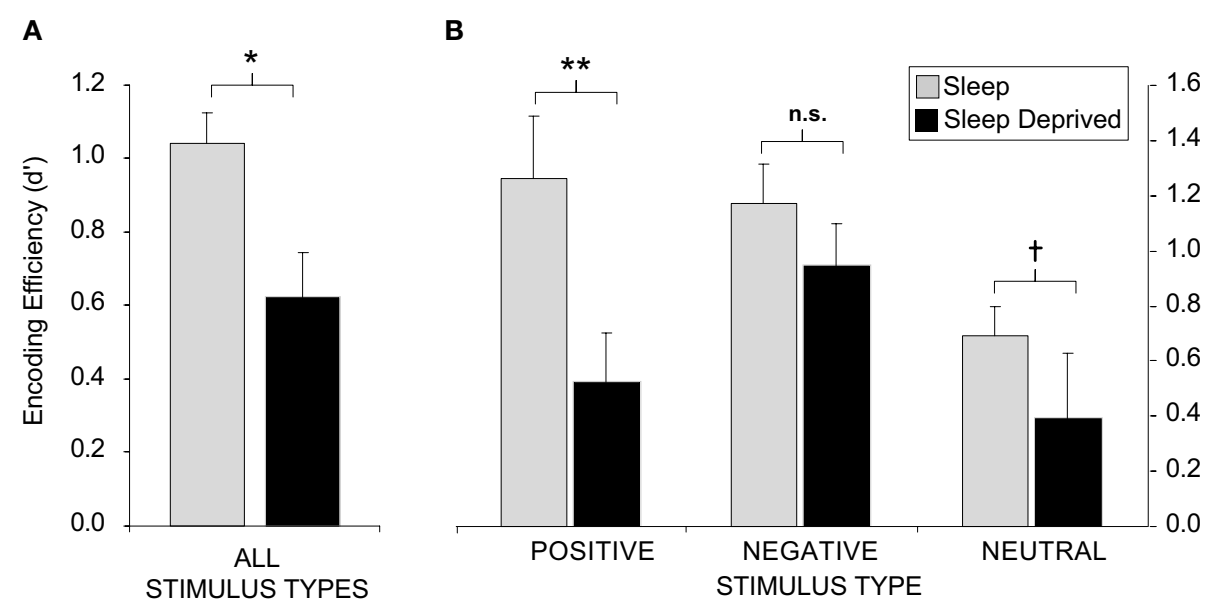

FIGURE 2 | Sleep deprivation and encoding of emotional and non-emotional declarative memory. Effects of $38 \mathrm{~h}$ of total sleep deprivation on encoding of human declarative memory $(\mathbf{A})$ when combined across all emotional and non-emotional categories; (B) When separated by emotional (positive and negative valence) and non-emotional (neutral valance) categories. When comparing between positive and negative words, within group, no significant difference was found for the sleep group while those that were deprived demonstrated greater recall for negative items $(p<0.05) .{ }^{\dagger} p<0.08,{ }^{*} p<0.05,{ }^{*} p<0.01$, error bars represent S.E.M.

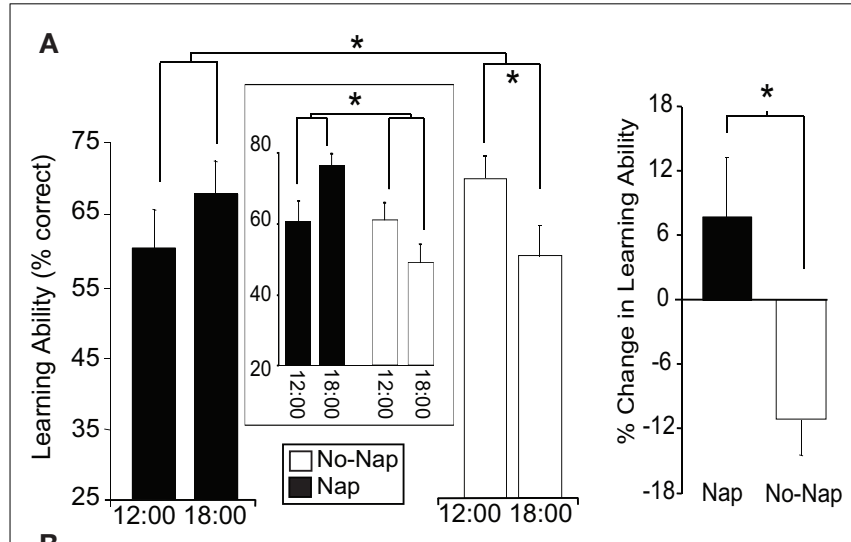

B
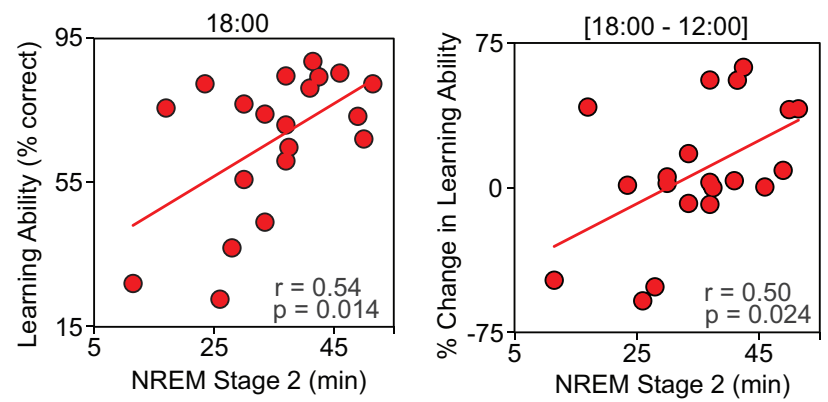

FIGURE 3 | (A) Episodic learning ability (\% face-name pairs encoded) in the nap and non-nap group at 12:00 and 18:00 (left), and the change in episodic learning ability between sessions (18:00-12:00; right). Episodic learning ability in a subset of nap and no-nap subjects ( $n=10$ per group), matched on initial 12:00 performance, is presented in the center box. Comparisons (line across bars) in both plots reflect significance* at: $p<0.05$. Error bars represent S.E.M. (B) Within the nap group, correlations with stage-2 NREM sleep and episodic learning ability at 12:00 (left-panel), 18:00 (middle-panel), and the change in learning ability between session (18:00-12:00; right-panel). (C)
C
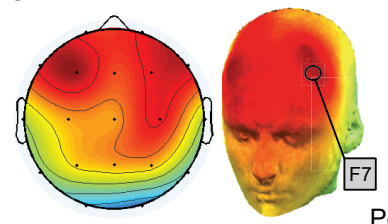

D

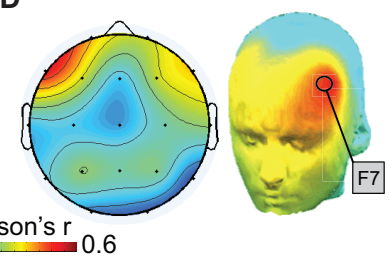

E

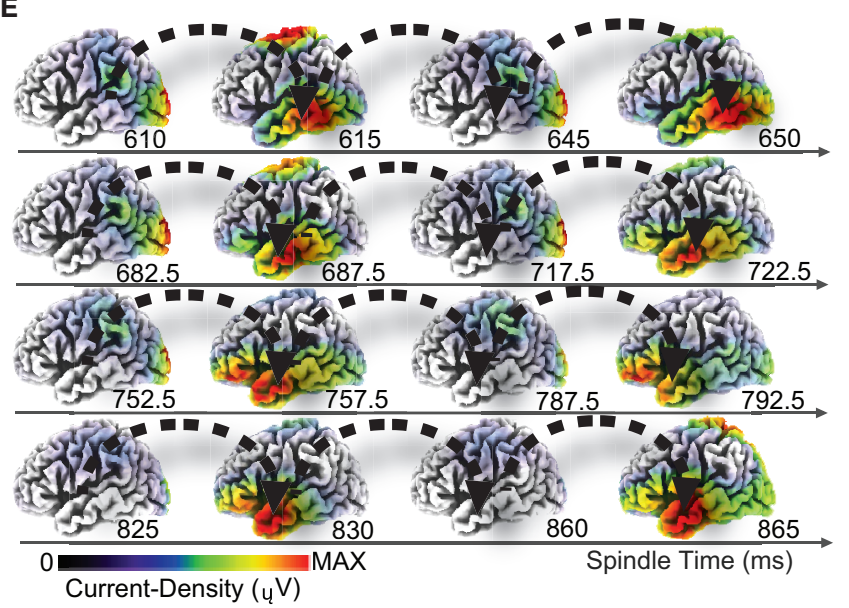

Topographic correlations (color-bar indicates Pearson's correlation strength) in the nap group between fast sleep spindles and episodic learning ability at 18:00 (post-nap), significant in derivations F3, F4, Fz, F7, F8, Fp1, and Fp2 over PFC, maximal at F7 ( $r=0.536, p=0.018)$. (D) Of these derivations, the change in episodic learning ability (18:00-12:00), significantly and conjointly correlated with fast spindles at derivation F7 over left PFC $(r=0.535$,

$p=0.018$ ). (E) sLORETA source time-series of fast sleep spindles identified by onset at $\mathrm{F}$, demonstrating a current-density loop recurring in left temporal lobe, proceeding the peak of the spindle. Modified from Mander et al. (2011). 
timing (both clock and circadian) of learning and testing constant between conditions.

\section{SLEEP AFTER LEARNING, FOR MEMORY CONSOLIDATION}

A robust and consistent literature has demonstrated the need for sleep after learning in the subsequent consolidation of hippocampal-dependent memory (Gais et al., 2002; Graves et al., 2003; Rauchs et al., 2004; Schabus et al., 2004; Fischer et al., 2005; Walker et al., 2005; Clemens et al., 2006; Ellenbogen et al., 2006; Ferrara et al., 2006; Fogel and Smith, 2006; Gais et al., 2006; Marshall et al., 2006; Wagner et al., 2006; Gais et al., 2007; Rasch et al., 2007; Alvarenga et al., 2008; Axmacher et al., 2008; Backhaus et al., 2008; Wilhelm et al., 2008; Landsness et al., 2009; Ramadan et al., 2009; Rudoy et al., 2009; Poe et al., 2010; Wamsley et al., 2010). In humans, arguably the first experimental description of a beneficial role of sleep for memory stabilization was provided by Jenkins and Dallenbach (1924), indicating a protective benefit of sleep (after approximately $4 \mathrm{~h}$ ) in preventing the normal decay-curve of forgetting that develops across time spent awake.

Building on these seminal findings, Ellenbogen et al. (2006), have since revealed the extent of sleep's ability to protect declarative memories from forgetting by systematically manipulating interference, employing an " $\mathrm{A}-\mathrm{B}-\mathrm{A}-\mathrm{C}$ " paradigm. In this paradigm, participants first learned unrelated word-pair associates, designated as list A-B (e.g., leaf-wheel etc.). After sleep at night, or wakefulness during the day, half of the subjects in each group learned a new, interfering list containing a new associate paired with the first word, designated as list A-C (e.g., leaf-nail etc), before being tested on the original A-B list (e.g., leaf-wheel etc). In the groups that did not experience the interfering challenge - simply being trained and then tested on list A-B - sleep provided a modest benefit to memory recollection (Figure 4). However, when testing the groups that were exposed to interfering list learning (list A-C) prior to recalling the original list (list A-B), a large and significant protective benefit was seen in those that slept (Figure 4). Therefore, sleep conferred a consolidation benefit that rendered recently encoded episodic memories resistant to the potentially aggravated effects of new learning of somewhat overlapping (hence potentially competing) memory representations the next day. Yet, it was only by using an interfering challenge, the A-C list, that the true benefit of sleep's protection of memory was revealed; a benefit that would not necessarily have been evident in a standard study-test memory paradigm. Such evidence would favor a mechanism by which sleep either (1) solidified the original (A-B) hippocampal representations, forcing encoding of related representations $(\mathrm{A}-\mathrm{C})$ in non-overlapping hippocampal networks, or (2) the transformation of the original (A-B) representations to a less hippocampally dependent state, allowing for hippocampal encoding of related new $(\mathrm{A}-\mathrm{C})$ representations that overlap with those previously coding the original $(\mathrm{A}-\mathrm{B})$ representations, but without detrimental interference.

Several reports by Born and colleagues have shown offline improvement on hippocampus-dependent memory following post-learning sleep attributed to early night sleep, rich in SWS (Diekelmann and Born, 2010). Evidence for such an effect comes first from studies demonstrating that partial deprivation of early SWS-rich sleep impairs recall of memories learned prior to sleep

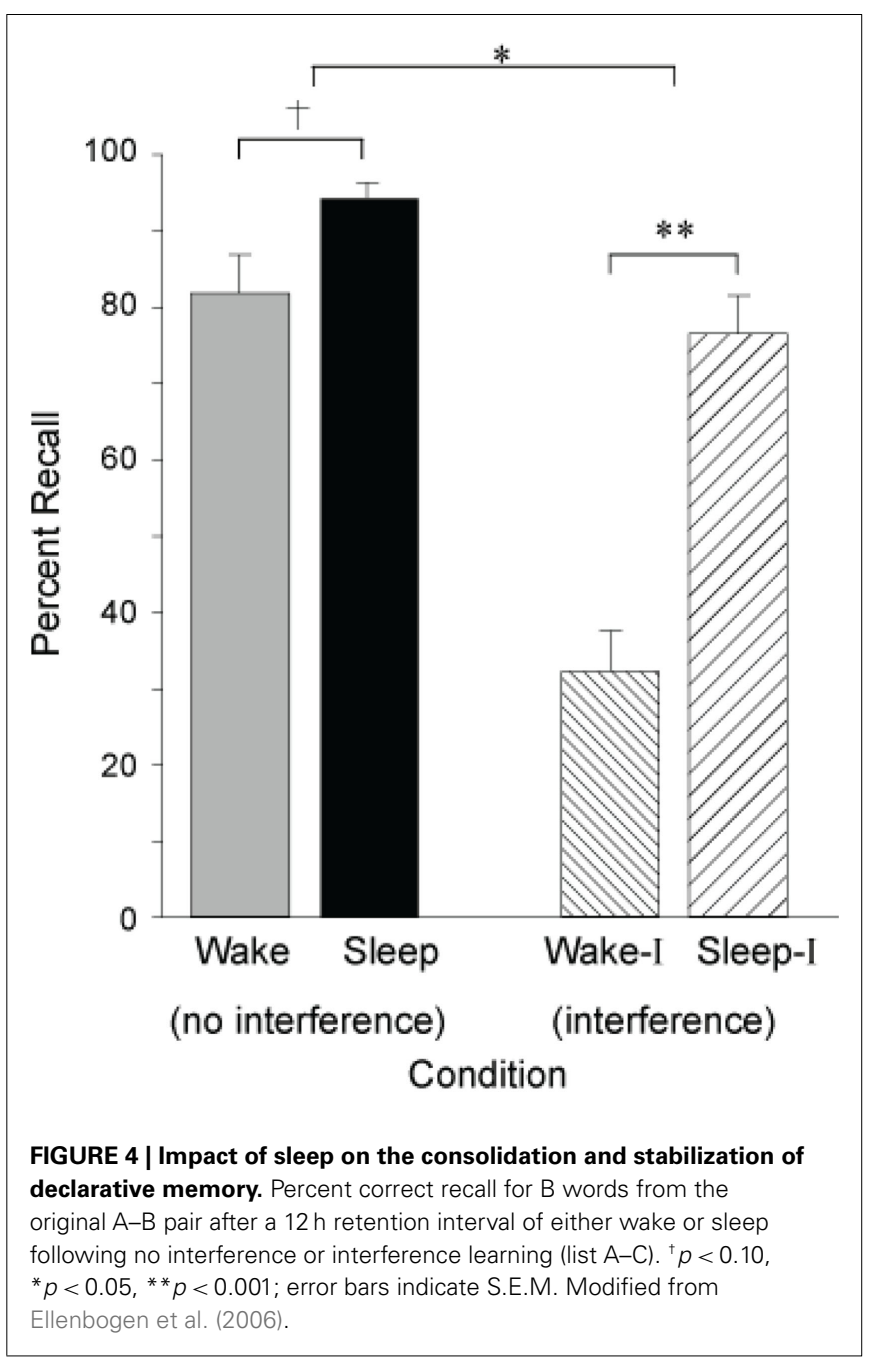

(Gais et al., 2000; Wagner et al., 2001; Wagner et al., 2002; Gais and Born, 2004; Fischer et al., 2011). The same group has also recently demonstrated a causal role for the slow cortical oscillation $(<1 \mathrm{~Hz})$ of NREM sleep in beneficially enhancing the consolidation of declarative memories by increasing activity in this frequency range. Following learning of a word-pair list, transcranial direct current stimulation was applied over the prefrontal cortex of participants during early night SWS, inducting slow oscillation-like field potentials (in this case, at $0.75 \mathrm{~Hz}$; Marshall et al., 2004, 2006). Consequently, a greater benefit on overnight retention was observed for the set of information learned prior to sleep. Direct current stimulation not only increased the amount of slow oscillation activity, as well as sleep spindle frequency activity, during the simulation period (and for some time after), but also enhanced next-day word-pair retention. These findings were interpreted in the context of slow oscillations potentially triggering spindle-regulated plasticity in cortex.

One mechanism proposed to underlie the benefit of sleep on hippocampal-dependent learning is the reactivation of previously encoded hippocampal memory representations during subsequent NREM sleep. In animals, the signature firing patterns of these hippocampal (as well as cortical) networks expressed 
during waking performance of spatial tasks and novel experiences appear to be "replayed" during subsequent NREM sleep (and in some studies, also REM; Wilson and McNaughton, 1994; Skaggs and McNaughton, 1996; Dave et al., 1998; Dave and Margoliash, 2000; Poe et al., 2000; Louie and Wilson, 2001; Ribeiro and Nicolelis, 2004; Jones, 2005; Ji and Wilson, 2007). Extending these findings, related evidence has been reported in the human brain using a virtual maze task in combination with positron emission tomography (PET) scanning (Peigneux et al., 2004). Daytime learning was initially associated with hippocampal activity. Then, during post-training sleep, there was a re-emergence of hippocampal activation, specifically during SWS. Most compelling, however, the amount of SWS reactivation in the hippocampus was proportional to the amount of next-day task improvement, suggesting that this reactivation is associated with offline memory improvement.

Building on the framework that memories, particularly those involving the hippocampus, are reactivated at night during sleep, recent work has taken advantage of the classical psychology effect of cue-dependent recall, and translated it into a sleep-dependent consolidation paradigm Rasch et al. (2007). Following learning of a spatial memory task that was paired with a rose sent cue, the same rose odor was re-presented during subsequent SWS that night - a time when consolidation was presumed to be occurring. Relative to a control condition where the odor was not presented again during SWS, the re-perfusion of the rose scent at night resulted in significantly improved recall the following day (when recall occurred without any odor cue). Moreover, the representation of the odor during SWS when previously paired with learning during encoding resulted in greater (re)activation of the hippocampus during SWS, as measured with fMRI. These findings support a role for SWS in the consolidation of declarative memory in relation to a prior context (here, odor), and may indicate an active reprocessing of initially hippocampal-dependent information during SWS. Building on these findings, Rudoy et al. (2009) have recently demonstrated similar sleep-dependent reactivation of memory using auditory rather than olfactory cues, describing a selective ability to manipulate individual item memory consolidation during NREM.

Determining the neural mechanisms that promote sleepdependent human memory consolidation remains an active topic of research, and debate. It is perhaps unlikely that multiple different memory systems, involving diverse cortical and/or subcortical networks, require the same underlying neural mechanisms for their modulation. Even if they do, it is not clear that this process would rely on just one type of sleep-stage physiology. Multiple models of sleep-dependent memory have been offered to account for the overnight facilitation of recall, which build on different aspects of neural activity during sleep. A systemslevel model of sleep-dependent memory processing (for discussion of a homeostatic cortical model of sleep-regulated plasticity, see Tononi and Cirelli, 2003) can be considered that involves reciprocal hippocampal-neocortical communication, with potential benefits not only for offline consolidation, but also renewed hippocampal encoding capacity upon awakening.

Within this framework (Marr, 1971; Squire and Alvarez, 1995; Squire et al., 2004; Frankland and Bontempi, 2005), the hippocampus initially binds cortical elements of an experience, creating a holistic episodic memory by way of hippocampal-cortical connections (Figure 5). Over time, and by way of iterative offline processes, increasing cortico-cortical connections develop, associated with decreasing dependence on the hippocampus. Therefore, the classical model of memory consolidation holds that neocortical structures become increasingly important for the representation of consolidated episodic memories, while the corresponding contribution of the hippocampus progressively decreases. In addition to its role in binding distributed cortical memory components, the hippocampus plays a critical role in reactivating these networks, specifically during sleep. This process of reactivation, assumed to occur over multiple sleep cycles across a night and/or multiple occurrences of sleep over many nights, is proposed to gradually strengthen the initially weak connections between neocortical sites, thereby reinforcing them. Eventually, this strengthening is suggested to allow the original information to be engaged in the cortex, largely independent of the hippocampus.

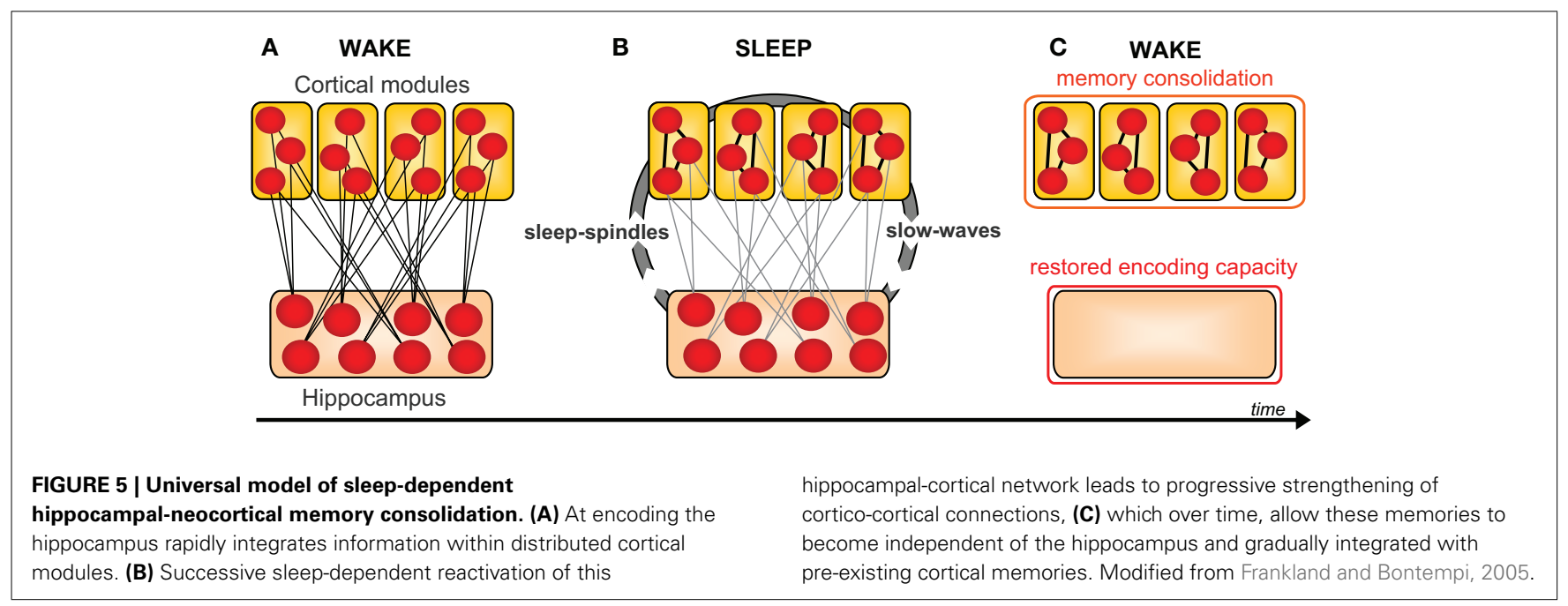


It has been posited that such a sleep-dependent process can offer two symbiotic benefits (Walker, 2009). The first is that episodic memories from the day prior should be more resistant to interference from new hippocampal learning the next day, due to the increased cortico-cortical connections formed during overnight consolidation (Figure 5). It is precisely this behavioral effect that was reported in the study by Ellenbogen et al. (2006), showing greater post-sleep resistance to interference, using the A-B - A$\mathrm{C}$ paradigm. In addition to consolidation, however, the second suggested benefit of this sleep-dependent dialog is the post-sleep reinstatement of sparse encoding capacity within the hippocampus, restoring the efficient ability for renewed next-day episodic encoding (Figure 5). This second premise appears to accurately explain the findings previously discussed describing a reduction in hippocampal encoding ability without intervening sleep (Yoo et al., 2007a; Van Der Werf et al., 2009), and conversely, the restoration of hippocampal-dependent learning following the presence of sleep (Mander et al., 2011).

Several reports have provided evidence supportive of this sleepdependent dialog and neural transformation of declarative memory. In the first such report, Takashima et al. (2006) examined the benefit of daytime naps on episodic declarative memory consolidation. In addition to a long-term evaluation of memory over 3 months, there was also a short-term evaluation of memory across the first day, which included an intervening nap period (90 $\mathrm{min})$ between training and testing of the original studied ("remote") stimuli. The duration of NREM SWS during the intervening nap correlated positively with later recognition memory performance, yet negatively with retrieval-related activity in the hippocampus, consistent with the notion of episodic representations becoming less dependent on the hippocampus, post-sleep. Extending these findings, Maquet and colleagues have since demonstrated that one night of post-training sleep deprivation, even following recovery sleep, significantly compromises these hippocampal-neocortical neural dynamics and associated memory recollection (Gais et al., 2007).

Beyond the contribution of NREM slow waves, a number of reports have described an association between NREM sleep spindle events and memory, including hippocampal-dependent learning. These short $(\sim 1 s)$ synchronous bursts of activity are expressed in the EEG in the $11-15 \mathrm{~Hz}$ frequency range (Sejnowski and Destexhe, 2000; Steriade, 2001; Smith et al., 2004), and coincide with hippocampal sharp waves and ripples (Siapas and Wilson, 1998; Sirota et al., 2003; Clemens et al., 2007, 2011; Mölle et al., 2009; Diekelmann and Born, 2010), possibly reflecting reactivation of learned memory representations (Mölle et al., 2006; Born, 2010; O’Neill et al., 2010).

Supporting a role for spindles in episodic memory processing, Gais et al. (2002), have shown significantly higher sleep spindle density following daytime episodic learning session (encoding of word-pair associates). Moreover, spindle density was associated with next day memory recall. These findings mirror observations by Meier-Koll et al. (1999), who reported a similar increase in spindles following learning of a hippocampally dependent maze task, and by Clemens et al. (2005) who have since identified a correlation between spindle density and overnight verbal memory retention (although interesting, not memory for faces). That such results reflect spindle-related memory processing is supported by recent data demonstrating that the same cortical areas active during learning are also (re)activated during post-encoding sleep spindle events (Bergmann et al., 2012). This learning-dependent change in spindle-related activity extends that of use-dependent changes in EEG slow waves that may reflect homeostatic process (Kattler et al., 1994; Huber et al., 2006).

Continued evidence suggests that sleep spindles can be separated into two subtypes based on frequency: "slow" (11-13 Hz) and "fast" (13-15 Hz; Werth et al., 1997; Zeitlhofer et al., 1997). The relevance of this separation from a memory consolidation perspective is highlighted by neuroimaging findings demonstrating that fast spindles are associated with, amongst other regions, significantly greater activation within the hippocampal complex (Schabus et al., 2007). Moreover, recent fMRI data have demonstrated that the occurrence of fast sleep spindles coincide with moments of increased functional connectivity between the hippocampus and areas of neocortex (Andrade et al., 2011), supporting a postulated component of the hippocampal-neocortical model of sleepdependent memory consolidation. Indeed, a growing number of reports describe select associations between episodic memory and fast- but not slow-spindle activity (Tamaki et al., 2008; Mander et al., 2011; Saletin et al., 2011; van der Helm et al., 2011). Nevertheless, the role of specific spindle frequency subtypes, and their local topographic influence, in hippocampal- and extrahippocampal-dependent memory processing represents a target for future research, with early discriminatory findings beginning to emerge (van der Helm et al., 2011). Secondly, while the role for sleep spindles in declarative memory processing is increasingly clear, there remains the need to dissociate which feature or combination of features of the spindle oscillation govern these memory processing advantages (e.g., number Clemens et al., 2005, density Saletin et al., 2011, or amplitude/power Schabus et al., 2008).

A consensus on the differential role of slow waves and spindles has also yet to be reached, with reports implicating sleep spindles (e.g., Gais et al., 2002; Schabus et al., 2004; Clemens et al., 2006; Nishida and Walker, 2007; Genzel et al., 2009; Saletin et al., 2011) or slow waves (e.g., Marshall et al., 2006; Takashima et al., 2006; Backhaus et al., 2007; Landsness et al., 2009) in memory processing. However, what, if any, inter-relationships exist between spindles and slow waves remains largely uncharacterized (Molle et al., 2011) and will require studies capable of distinguishing between spindle-driven and slow wave-driven memory mechanisms, or identifying their combined influence. Moreover, the potential role for REM sleep and its associated physiological features in declarative memory processing remains relevant (Rauchs et al., 2004; Walker, 2009, see below discussion). By way of such future investigations, an increasingly nuanced characterization of dissociable sleep-dependent memory mechanisms can be reached.

While evidence reviewed above focuses on NREM sleep in declarative memory consolidation, REM sleep should not be discounted. REM sleep may be especially critical for processing of emotionally salient memories. Early work reported that the overnight retention of emotional details of a narrative story, relative to emotionally neutral details, was superior following latenight sleep (a time period rich in REM sleep; Wagner et al., 2001). It 
has subsequently been demonstrated that the speed of recognizing emotional face expressions presented prior to sleep is significantly improved the next day, the amount of which positively correlated with the amount of intervening REM sleep (Wagner et al., 2007). Moreover, not only does the amount of time and speed of entry into REM sleep predict the degree of subsequent strengthening and hence offline consolidation of emotional (and not neutral) memory, but specifically the amount of REM sleep theta EEG activity $(4-7 \mathrm{~Hz})$ expressed over the prefrontal cortex that predicts memory retention (Nishida et al., 2009). These findings have lead to the proposal that REM sleep represents a neurobiological brain-state particularly amenable to emotional memory processing (Pare et al., 2002; Hu et al., 2006; Walker, 2009; Walker and van der Helm, 2009), with theta oscillations proposed as a carrier frequency that potentially allows disparate brain regions that initially encode information to selectively interact offline. By doing so, REM sleep theta may afford the ability to strengthen distributed aspects of specific memory representations across related but different anatomical networks, and/or promote their integration into pre-existing autobiographical memory networks (Cahill, 2000; Jones and Wilson, 2005).

\section{CONSOLIDATION: SELECTIVE OR UNIVERSAL SLEEP BENEFITS?}

Evidence reviewed to this point implicates sleep in the consolidation and hence superior offline retention of declarative memory, relative to equivalent time awake. However, the capacity to forget can, in certain contexts, be as important as the need for memory retention, both in day-to-day life (e.g., forgetting last week's parking spot in preference for today's), and clinically (e.g., post-traumatic stress disorder and addiction). Considered to be adaptive, selective forgetting has been shown to decrease neural resources required for targeted remembering (Levy and Anderson, 2002; Kuhl et al., 2007), and as a consequence, may afford improved efficiency of subsequent recall of select information (Block, 1971; Anderson et al., 2004; Levy and Anderson, 2008). Building on such evidence, here we offer the thesis that the goal of offline sleep-dependent memory processing is not the verbose, ubiquitous, and non-selective consolidation of all information recently encoded. Nor is it the generalized weakening of representations (Crick and Mitchison, 1983). Instead, we suggest offline sleep-dependent memory processing may involve a discriminatory mechanism, affording a balance of retention, and forgetting, with item selection determined by salience cues present during wake, such as novelty, emotionality, reward value, and explicit conscious instruction (Figure 6).

Emerging reports have begun to support this more nuanced, discriminatory framework of sleep-dependent memory processing. Fitting with such prior section, sleep (Hu et al., 2006; Atienza and Cantero, 2008), and specifically REM sleep (Wagner et al., 2001; Nishida et al., 2009), has been demonstrated to target the selective consolidation of emotional relative to nonemotional experiences. Moreover, sleep appears capable of separating episodic experiences into component parts, preferentially consolidating those of greatest affective salience (Payne et al., 2008). Beyond emotion, sleep has recently been demonstrated to selectively enhance information based on waking knowledge of potential monetary reward prior to sleep (Fischer and Born, 2009), even when such knowledge of a reward was announced after initial learning (but before sleep). Further, the mere knowledge that some learned items will be subject to later post-sleep testing, presumably involving a difference in salient relevance, amongst other things, can selectively increase the offline sleep-dependent consolidation benefit (Wilhelm et al., 2011).

Explicit conscious instruction at the time of encoding also appears to represent a powerful modulatory influence on subsequent sleep-dependent consolidation. Using a directed forgetting paradigm, the impact of cued instruction during learning to

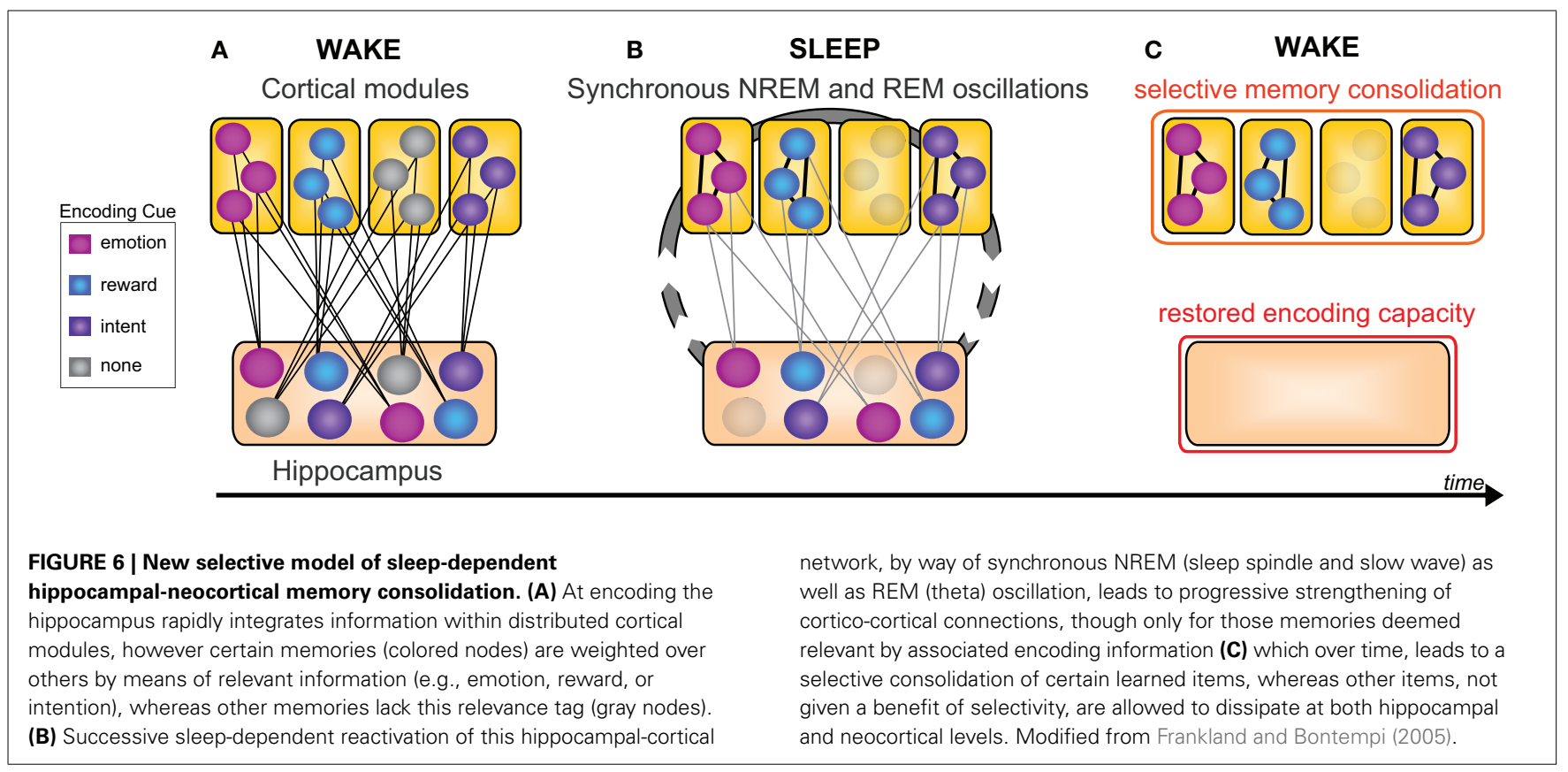


either remember or forget, prior to sleep, on subsequent differential retention of memory after sleep has recently been examined (Saletin et al., 2011). Relative to time awake, sleep subsequently and selectively ignored the facilitation of items previously cued to be forgotten, yet preferentially enhance recall for items cued to be remembered (Figure 7A). This effect was further characterized as a difference-score (remember words - forget words). This difference measure, reflecting the efficiency of accomplishing targeted remembering and forgetting, demonstrating a greater separation between recall of remember relative to forget items following sleep (Figure 7B). Thus, sleep did not benefit all memories universally, instead being selective for those items cued for remembering and not those cued to be forgotten, indicative of specificity based on prior waking instruction. Moreover, the magnitude of this directed forgetting effect was positively predicted by fast spindles over left parietal cortex, suggesting their potential role in differential "gating" of offline consolidation, governing the directional fate of each memory item class (remember, forget; Figure 7C). Additionally, the same parietal fast spindles differentially and bi-directionally modulated each word class individually; positively predicting recall for remember words alone while, conversely, negatively predicting recall for forget words (Figure 7D,E). This evidence favors a sleep mechanism that not only promotes remembering but also actively instigates forgetting. Offering deeper anatomical insights, EEG source analyses revealed an loop of activity occurring during these parietal fast spindle events, consisting of areas previously implicated in differential remembering and forgetting memory (Wylie et al., 2008): prefrontal, medial temporal, and posterior parietal cortices. Such a network may support memory modulation whereby "top-down" cues of instructed intent (prefrontal cortex; remember, forget) are coordinated and utilized during offline processing of "bottom-up" item memory (medial temporal lobe), and potentially integrated into association regions (parietal cortex) for consolidation (Shimamura, 2011). Related findings have

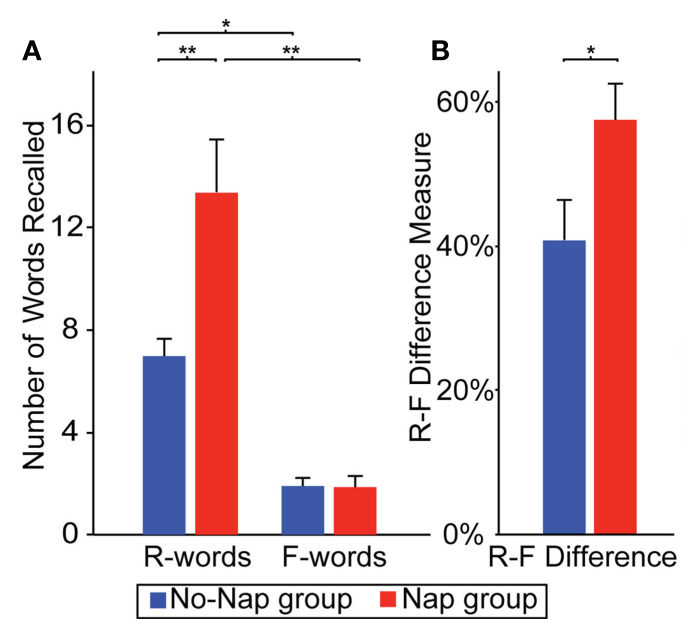

D

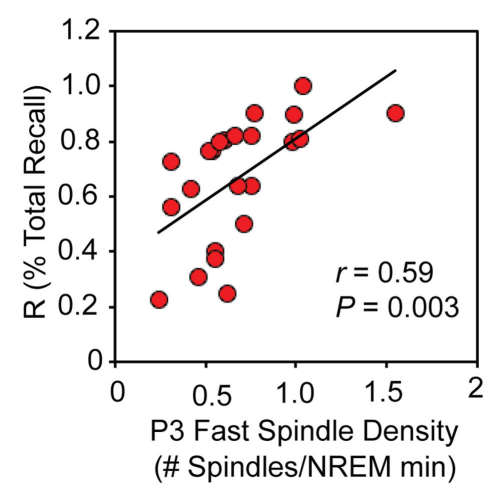

FIGURE 7 | Role of sleep on directed remembering and forgetting.

(A) Memory performance: Number of words recalled based on prior cue instruction (Remember, R-words; Forget, F-words) in the nap and no-nap groups and the (B) efficiency measure of directed forgetting, calculated as the subtraction of these scores (R-F; expressed as a proportion of total recall). Between group comparisons (line across bars) reflect significance at: ${ }^{*} p<0.05$ and ${ }^{* *} p<0.01$. Error bars represent S.E.M. (C-E) Physiological data. Relationship between memory performance

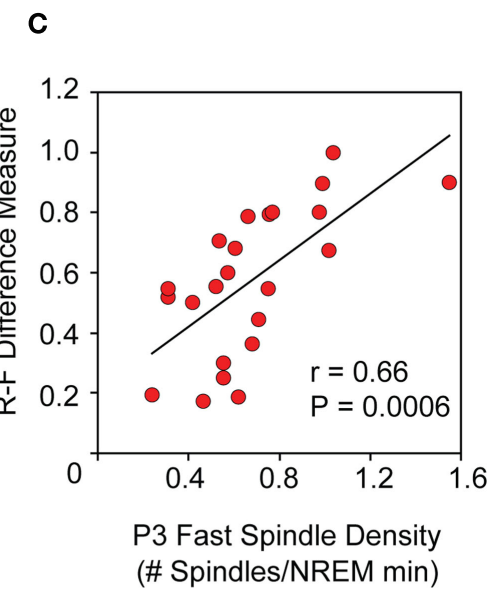

$\mathbf{E}$

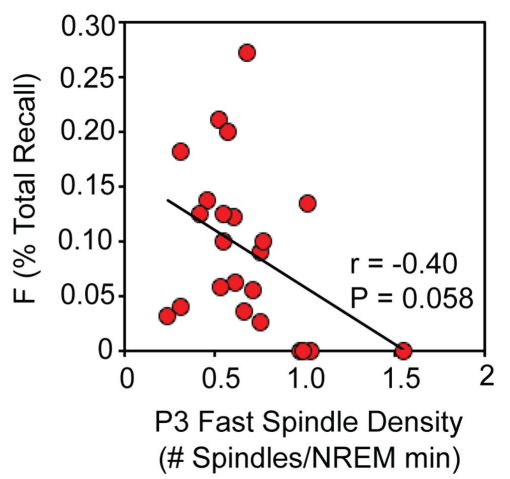

and sleep spindles: (C) Scatter-plot and linear regression of the relationship between fast sleep-spindle density at P3 and the R-F score across participants in the nap group. (C-D) Scatterplots demonstrating that the same P3 fast spindles (D) positively predict R-words and (E) negatively predict F-words, though neither correlation is as strong as the $\mathrm{R}-\mathrm{F}$ difference measure, suggesting a relative selectivity of sleep-dependent memory consolidation. Modified from Saletin et al. (2011). 
been reported using an episodic memory paradigm involving the intentional suppression and contemplation of word items prior to sleep, with those intentionally suppressed during initial exposure showing significantly less offline sleep-dependent memory benefit (Fischer et al., 2011). Adding to these findings, post-encoding sleep deprivation can further impair the subsequent offline development of these differential remember and forget consolidation effects, dependent, in part, on hippocampal signals at the time of encoding (Rauchs et al., 2011).

It is of note that the presentation of olfactory cues during sleep for memory reactivation described earlier only resulted in facilitated overnight consolidation when the same odor was initially presented at the time of learning (Rasch et al., 2007). Odor presented during sleep in isolation, without prior waking association with learning, conferred neither an overnight memory consolidation advantage nor elevated hippocampal reactivation during sleep. This finding appears consistent with the proposed model of selective memory consolidation, suggesting that even the success of memory reactivation during sleep critically depends on associating those memories with the cues during initial encoding, prior to sleep. Such associations would effectively label specific memory representations at the time of learning with contextually salient tags; offering the capacity for selective reactivation, and subsequent consolidation, during sleep.

A further circumstance that may determine selective memory consolidation is the relationship between newly learned representations and pre-existing autobiographical memory schemata (Lewis and Durrant, 2011). Experiences that hold relevance to the organism's autobiographical past have the potential for being registered, as a consequence, with superior salience, and hence undergo preferential overnight consolidation. Similarly, employing learning strategies such as mnemonic techniques that assist in elaboration on, and integration amongst, new learning (or even pre-existing information; Wang and Thomas, 2000) may similarly promote selective offline consolidation during sleep.

When taken together, this collection of studies suggest that sleep does not universally consolidate all episodic memories learned during the prior day(s). Instead, sleep appears to be more ecologically attuned to qualitative aspects of encoded experiences at the time of initial learning e.g. emotionality (Hu et al., 2006; Wagner et al., 2006, 2007; Payne et al., 2008; Nishida et al., 2009; Walker and van der Helm, 2009; Payne and Kensinger, 2011), reward potential (Fischer and Born, 2009; Wilhelm et al., 2011), or explicit cue instructions and intentions (Fischer et al., 2011; Rauchs et al., 2011; Saletin et al., 2011), resulting in discriminatory offline memory processing. Such findings additionally offer key anatomical targets for the understanding of sleep-dependent memory processing, focusing on those regions whose interactions with the medial temporal lobe support selective memory processing e.g., amygdala for emotional memories (Hu et al., 2006; Yoo et al., 2007b; Nishida et al., 2009; Walker and van der Helm, 2009; Payne and Kensinger, 2011), striatum for reward signals (Knutson and Adcock, 2005) and the prefrontal cortex for directed intentional cues (Anderson et al., 2004; Wylie et al., 2008; Rauchs et al., 2011). Parenthetically, this selective sleep benefit, including the bi-directional influence of spindles on remembering and forgetting, seems difficult to reconcile within simple opportunistic frameworks of offline consolidation (Mednick et al., 2011). Instead, such evidence appears to warrant a shift in perspective from notions of sleep affording a universal consolidation benefit to recently encoded information (Figure 4), to one in which unique and specific sleep-stage physiologies select item information for preferential retention, based on prior waking cues (Figure 6); a process conceptually similar to a sleep-slave consolidation system, governed by prior waking cues, and yielding discriminatory long-term memory consolidation.

While remaining speculative, potentially novel clinical and therapeutic implications stem from this revised model of selective memory consolidation. Perhaps most prominent are the circumstance of anxiety disorders as well as major depression, both of which express dysfunction in memory as well as sleep abnormalities (Armitage, 1995; van Wingen et al., 2010; Dickie et al., 2011; Harvey, 2011). In major depression, disproportionate rumination of past negative autobiographical events can be prevalent, contributed to by the proposal of a bias in initial encoding of negative experiences (Taconnat et al., 2010; van Wingen et al., 2010). In the context of our model, this initial encoding bias may lead to the maladaptive further potentiation of those selectively negative experiences during sleep, the consequence of which would be their persistent dominance in long-term memory, further encouraging negative rumination. In anxiety disorders, including posttraumatic stress disorder and phobia, a similarly biased associative encoding of episodic experiences with strong negative aversive emotion (Mitte, 2008; Dickie et al., 2011) may tag those experiences for disproportional selective consolidation, potentiating the trauma experiences or phobic associations (and see Walker and van der Helm, 2009 for discussion of emotion processing of memory in PTSD). Conversely, however, these same mechanisms could be considered for use in therapeutic intervention. In both conditions, targeting the problematic memory sources during wake, by recollection and hence reactivation (Lee et al., 2004; Stickgold and Walker, 2005; Tronson and Taylor, 2007), can offer the capacity for developing new positive (rather than negative), or simply neutral, associations, pre-sleep. Subsequent selective consolidation of these new positive or neutral associations in sleep, put in place during prior wake, could help foster a revised and therapeutically advantageous collection of memory representations in the individual's autobiographical history. Finally, similar bi-directional notions of memory modulation may also be relevant in addiction, both in the maladaptive potentiation of addiction associations, but also their potential targeted reversal leading to extinction of acquired associations.

\section{CONCLUSIONS}

While not fully complete, a new taxonomy of sleep-dependent memory processing is emerging. This revised view describes a symbiotic role for sleep both before and after learning in optimally coordinating the initial encoding and subsequent consolidation of hippocampal-dependent memory, respectively. By way of such a mechanism, sleep appears capable of maximizing retention only of the most salient, relevant hippocampal memories, while forgetting (perhaps actively), those non-essential to the organism - an optimal algorithm for equilibrium within memory networks. 


\section{ACKNOWLEDGMENTS}

The author wishes to thank Edwin Robertson, Allison Harvey, Ronald Dahl, Els van der Helm, Bryce Mander, Stephanie Greer, Andrea Goldstein, Robert Stickgold, Ninad Gujar and for

\section{REFERENCES}

Alvarenga, T., Patti, C., Andersen, M., Silva, R., Calzavara, M., Lopez, G., Frussa-Filho, R., and Tufik, S. (2008). Paradoxical sleep deprivation impairs acquisition, consolidation, and retrieval of a discriminative avoidance task in rats. Neurobiol Learn Mem. 90, 624-632.

Anderson, M. C., Ochsner, K. N., Kuhl, B., Cooper, J., Robertson, E., Gabrieli, S. W., Glover, G. H., and Gabrieli, J. D. (2004). Neural systems underlying the suppression of unwanted memories. Science 303, 232-235.

Andrade, K. C., Spoormaker, V. I., Dresler, M., Wehrle, R., Holsboer, F., Samann, P. G., and Czisch, M. (2011). Sleep spindles and hippocampal functional connectivity in human NREM sleep. J. Neurosci. 31, 10331-10339.

Armitage, R. (1995). Microarchitectural findings in sleep EEG in depression: diagnostic implications. Biol. Psychiatry 37, 72-84.

Atienza, M., and Cantero, J. L. (2008). Modulatory effects of emotion and sleep on recollection and familiarity. J. Sleep Res. 17, 285-294.

Axmacher, N., Elger, C. E., and Fell, J. (2008). Ripples in the medial temporal lobe are relevant for human memory consolidation. Brain 131, 1806-1817.

Backhaus, J., Born, J., Hoeckesfeld, R., Fokuhl, S., Hohagen, F., and Junghanns, K. (2007). Midlife decline in declarative memory consolidation is correlated with a decline in slow wave sleep. Learn. Mem. 14, 336-341.

Backhaus, J., Hoeckesfeld, R., Born, J., Hohagen, F., and Junghanns, K. (2008). Immediate as well as delayed post learning sleep but not wakefulness enhances declarative memory consolidation in children. Neurobiol. Learn. Mem. 89, 76-80.

Bergmann, T. O., Molle, M., Diedrichs, J., Born, J., and Siebner, H. R. (2012). Sleep spindle-related reactivation of category-specific cortical regions after learning face-scene associations. NeuroImage 59, 2733 2742.

Block, R. (1971). Effects of instructions to forget short-term memory. J. Exp. Psychol. 89, 9.
Born, J. (2010). Slow-wave sleep and the consolidation of long-term memory. World J. Biol. Psychiatry 11(Suppl. 1), 16-21.

Cahill, L. (2000). Neurobiological mechanisms of emotionally influenced, long-term memory. Prog. Brain Res. 126, 29-37.

Cahill, L., and McGaugh, J. L. (1998). Mechanisms of emotional arousal and lasting declarative memory. Trends Neurosci. 21, 294-299.

Clemens, Z., Fabo, D., and Halasz, P. (2005). Overnight verbal memory retention correlates with the number of sleep spindles. Neuroscience 132, 529-535.

Clemens, Z., Fabó, D., and Halász, P. (2006). Twenty-four hours retention of visuospatial memory correlates with the number of parietal 52-56.

Clemens, Z., Molle, M., Eross, L., Barsi, P., Halasz, P., and Born, J. (2007). Temporal coupling of parahippocampal ripples, sleep spindles and slow oscillations in humans. Brain 130, 2868-2878.

Clemens, Z., Mölle, M., Eross, L., Jakus, R., Rásonyi, G., Halász, P., and Born, J. (2011). Fine-tuned coupling between human parahippocampal ripples and sleep spindles. Eur. J. Neurosci. 33, 511-520.

Crick, F., and Mitchison, G. (1983). The function of dream sleep. Nature 304, 111-114.

Dave, A. S., and Margoliash, D. (2000). Song replay during sleep and computational rules for sensorimotor vocal learning. Science 290, 812-816.

Dave, A. S., Yu, A. C., and Margoliash, D. (1998). Behavioral state modulation of auditory activity in a vocal motor system. Science 282, 2250-2254.

Dickie, E. W., Brunet, A., Akerib, V., and Armony, J. L. (2011). Neural traumatic stress disorder: a longitudinal fMRI investigation of memory encoding. Neuropsychologia 49, 1771-1778.

Diekelmann, S., and Born, J. (2010). The memory function of sleep. Nat. Rev. Neurosci. 11, 114-126.

Drummond, S. P., Brown, G. G., Gillin, J. C., Stricker, J. L., Wong, E. C., and Buxton, R. B. (2000). Altered brain response to verbal learning following sleep deprivation. Nature 403, 655-657. sleep spindles. Neurosci. Lett. 403, correlates of recovery from post-

thoughtful insights. This work was supported in part by grants from the National Institutes of Health (NIA AG31164 to Matthew P. Walker); the National Science Foundation (GRFP to Jared M. Saletin); and the University of California, Berkeley.

Drummond, S. P., Gillin, J. C., and Brown, G. G. (2001). Increased cerebral response during a divided attention task following sleep deprivation. J. Sleep Res. 10, 85-92.

Ellenbogen, J. M., Hulbert, J. C. Stickgold, R., Dinges, D. F., and Thompson-Schill, S. L. (2006). Interfering with theories of sleep and memory: sleep, declarative memory, and associative interference. Curr. Biol. 16, 1290-1294.

Ferrara, M., Iaria, G., De Gennaro, L. Guariglia, C., Curcio, G., Tempesta, D., and Bertini, M. (2006). The role of sleep in the consolidation of route learning in humans: a behavioural study. Brain Res. Bull. 71, 4-9.

Fischer, S., and Born, J. (2009). Anticipated reward enhances offline learning during sleep. J. Exp. Psychol. Learn Mem. Cogn. 35, 1586-1593.

Fischer, S., Diekelmann, S., and Born, J. (2011). Sleep's role in the processing of unwanted memories. J. Sleep Res. 20, 267-274.

Fischer, S., Nitschke, M., Melchert, U., Erdmann, C., and Born, J. (2005). Motor memory consolidation in sleep shapes more effective neuronal representations. J. Neurosci. 25 , 11248-11255.

Fogel, S., and Smith, C. (2006). Learning-dependent changes in sleep spindles and Stage 2 sleep. J. Sleep Res. 15, 250-255.

Frankland, P. W., and Bontempi, B. (2005). The organization of recent and remote memories. Nat. Rev. Neurosci. 6, 119-130.

Gais, S., Albouy, G., Boly, M., DangVu, T. T., Darsaud, A., Desseilles, M., Rauchs, G., Schabus, M., Sterpenich, V., Vandewalle, G., Maquet, P., and Peigneux, P. (2007). Sleep transforms the cerebral trace of declarative memories. Proc. Natl. Acad. Sci. U.S.A. 104, 18778-18783.

Gais, S., and Born, J. (2004). Declarative memory consolidation: mechanisms acting during human sleep. Learn. Mem. 11, 679-685.

Gais, S., Lucas, B., and Born, J. (2006). Sleep after learning aids memory recall. Learn. Mem. 13, 259-262.

Gais, S., Molle, M., Helms, K., and Born, J. (2002). Learning-dependent increases in sleep spindle density. $J$. Neurosci. 22, 6830-6834.

Gais, S., Plihal, W., Wagner, U., and Born, J. (2000). Early sleep triggers memory for early visual discrimination skills. Nat. Neurosci. 3, 1335-1339.

Genzel, L., Dresler, M., Wehrle, R. Grozinger, M., and Steiger, A. (2009). Slow wave sleep and REM sleep awakenings do not affect sleep dependent memory consolidation. Sleep 32, 302-310.

Graves, L., Heller, E., Pack, A., and Abel, T. (2003). Sleep deprivation selectively impairs memory consolidation for contextual fear conditioning. Learn. Mem. 10, 168-176.

Harrison, Y., and Horne, J. A. (2000). Sleep loss and temporal memory. Q. J. Exp. Psychol. A. 53, 271-279.

Harvey, A. G. (2011). Sleep and circadian functioning: critical mechanisms in the mood disorders? Annu. Rev. Clin. Psychol. 7, 297-319.

$\mathrm{Hu}$, P., Stylos-Allan, M., and Walker, M. P. (2006). Sleep facilitates consolidation of emotional declarative memory. Psychol. Sci. 17, 891-898.

Huber, R., Ghilardi, M. F., Massimini, M., Ferrarelli, F., Riedner, B. A., Peterson, M. J., and Tononi, G. (2006). Arm immobilization causes cortical plastic changes and locally decreases sleep slow wave activity. Nat. Neurosci. 9, 1169-1176.

Huijbers, W., Pennartz, C. M., Cabeza, R., and Daselaar, S. M. (2011) The hippocampus is coupled with the default network during memory retrieval but not during memory encoding. PLoS ONE 6, e17463. doi:10.1371/journal.pone.0017463

Jenkins, D. A., and Dallenbach, K. M. (1924). Obliviscence during sleep and waking. Am. J. Psychol. 35, 605-612.

Ji, D., and Wilson, M. (2007). Coordinated memory replay in the visual cortex and hippocampus during sleep. Nat. Neurosci. 10, 100-107.

Jones, B. E. (2005). From waking to sleeping: neuronal and chemical substrates. Trends Pharmacol. Sci. 26, 578-586.

Jones, M. W., and Wilson, M. A. (2005). Theta rhythms coordinate hippocampal-prefrontal interactions in a spatial memory task. PLoS Biol. 3, e402. doi:10.1371/journal.pbio.0030402

Kattler, H., Dijk, D. J., and Borbély, A. A. (1994). Effect of unilateral somatosensory stimulation prior to sleep on the sleep EEG in humans. $J$. Sleep Res. 3, 159-164. 
Knutson, B., and Adcock, R. A. (2005). Remembrance of rewards past. Neuron 45, 331-332.

Kuhl, B. A., Dudukovic, N. M., Kahn, I., and Wagner, A. D. (2007). Decreased demands on cognitive control reveal the neural processing benefits of forgetting. Nat. Neurosci. 10, 908-914.

Landsness, E. C., Crupi, D., Hulse, B. K., Peterson, M. J., Huber, R., Ansari, H., Coen, M., Cirelli, C., Benca, R. M., Ghilardi, M. F., and Tononi, G. (2009). Sleep-dependent improvement in visuomotor learning: a causal role for slow waves. Sleep 32, 1273-1284.

Lee, J. L. C., Everitt, B. J., and Thomas, K. L. (2004). Independent cellular processes for hippocampal memory consolidation and reconsolidation. Science 304, 839-843.

Levy, B. J., and Anderson, M. C. (2002). Inhibitory processes and the control of memory retrieval. Trends Cogn. Sci. (Regul. Ed.) 6, 299-305.

Levy, B. J., and Anderson, M. C. (2008). Individual differences in the suppression of unwanted memories: the executive deficit hypothesis. Acta Psychol. (Amst) 127, 623-635.

Lewis, P. A., and Durrant, S. J. (2011). Overlapping memory replay during sleep builds cognitive schemata. Trends Cogn. Sci. (Regul. Ed.) 15, 343-351.

Louie, K., and Wilson, M. (2001). Temporally structured replay of awake hippocampal ensemble activity during rapid eye movement sleep. $\mathrm{Neu}$ ron 29, 145-156.

Mander, B. A., Santhanam, S., Saletin, J. M., and Walker, M. P. (2011). Wake deterioration and sleep restoration of human learning. Curr. Biol. 21, R183-R184.

Marr, D. (1971). Simple memory: a theory for archicortex. Philos. Trans. R. Soc. Lond. B Biol. Sci. 262, 23-81.

Marshall, L., Helgadóttir, H., Mölle, M., and Born, J. (2006). Boosting slow oscillations during sleep potentiates memory. Nature 444, 610-613.

Marshall, L., Mölle, M., Hallschmid, M. and Born, J. (2004). Transcranial direct current stimulation during sleep improves declarative memory. J. Neurosci. 24, 9985-9992.

Mednick, S. C., Cai, D. J., Shuman, T., Anagnostaras, S., and Wixted, J. T. (2011). An opportunistic theory of cellular and systems consolidation. Trends Neurosci. 34, 504-514.

Meier-Koll, A., Bussmann, B., Schmidt, C., and Neuschwander, D. (1999). Walking through a maze alters the architecture of sleep. Percept. Mot. Skills 88, 1141-1159.

Mitte, K. (2008). Memory bias for threatening information in anxiety and anxiety disorders: a metaanalytic review. Psychol. Bull. 134, 886-911.

Molle, M., Bergmann, T. O., Marshall, L., and Born, J. (2011). Fast and slow spindles during the sleep slow oscillation: disparate coalescence and engagement in memory processing. Sleep 34, 1411-1421.

Mölle, M., Eschenko, O., Gais, S., Sara, S. J., and Born, J. (2009). The influence of learning on sleep slow oscillations and associated spindles and ripples in humans and rats. Eur. J. Neurosci. 29, 1071-1081.

Mölle, M., Yeshenko, O., Marshall, L., Sara, S. J., and Born, J. (2006). Hippocampal sharp wave-ripples linked to slow oscillations in rat slowwave sleep. J. Neurophysiol. 96, 62-70.

Morris, G. P., Williams, H. L., and Lubin, A. (1960). Misperception and disorientation during sleep deprivation. Arch. Gen. Psychiatry 2, 247-254.

Nishida, M., Pearsall, J., Buckner, R. L., and Walker, M. P. (2009). REM sleep, prefrontal theta, and the consolidation of human emotional memory. Cereb. Cortex 19, 1158-1166.

Nishida, M., and Walker, M. (2007). Daytime naps, motor memory consolidation and regionally specific sleep spindles. PLoS ONE 2, e341. doi:10.1371/journal.pone.0000341

O’Neill, J., Pleydell-Bouverie, B., Dupret, D., and Csicsvari, J. (2010). Play it again: reactivation of waking experience and memory. Trends Neurosci. 33, 220-229.

Pare, D., Collins, D. R., and Pelletier, J. G. (2002). Amygdala oscillations and the consolidation of emotional memories. Trends Cogn. Sci. (Regul. Ed.) 6, 306-314.

Payne, J. D., and Kensinger, E. A. (2011). Sleep leads to changes in the emotional memory trace: evidence from fMRI. J. Cogn. Neurosci. 23, 1285 1297.

Payne, J. D., Stickgold, R., Swanberg, K., and Kensinger, E. A. (2008). Sleep preferentially enhances memory for emotional components of scenes. Psychol. Sci. 19, 781-788.

Peigneux, P., Laureys, S., Fuchs, S., Collette, F., Perrin, F., Reggers, J., Phillips, C., Degueldre, C., Del Fiore, G., Aerts, J., Luxen, A., and Maquet, P. (2004). Are spatial memories strengthened in the human hippocampus during slow wave sleep? Neuron 44, 535-545.
Poe, G., Nitz, D., Mcnaughton, B. and Barnes, C. (2000). Experiencedependent phase-reversal of hippocampal neuron firing during REM sleep. Brain Res. 855, 176-180.

Poe, G. R., Walsh, C. M., and Bjorness, T. E. (2010). Both duration and timing of sleep are important to memory consolidation. Sleep 33, 1277-1278.

Ramadan, W., Eschenko, O., and Sara, S. J. (2009). Hippocampal sharp wave/ripples during sleep for consolidation of associative memory. PLoS ONE 4, e6697. doi:10.1371/journal.pone.0006697

Rasch, B., Büchel, C., Gais, S., and Born, J. (2007). Odor cues during slow-wave sleep prompt declarative memory consolidation. Science 315, 1426-1429.

Rauchs, G., Bertran, F., Guillery-Girard, B., Desgranges, B., Kerrouche, N. Denise, P., Foret, J., and Eustache, F. (2004). Consolidation of strictly episodic memories mainly requires rapid eye movement sleep. Sleep 27, 395-401.

Rauchs, G., Feyers, D., Landeau, B. Bastin, C., Luxen, A., Maquet, P. and Collette, F. (2011). Sleep contributes to the strengthening of some memories over others, depending on hippocampal activity at learning. $J$. Neurosci. 31, 2563-2568.

Ribeiro, S., and Nicolelis, M. (2004). Reverberation, storage, and postsynaptic propagation of memories during sleep. Learn. Mem. 11, 686-696.

Rudoy, J. D., Voss, J. L., Westerberg, C. E., and Paller, K. A. (2009). Strengthening individual memories by reactivating them during sleep. Science $326,1079$.

Saletin, J. M., Goldstein, A. N., and Walker, M. P. (2011). The role of sleep in directed forgetting and remembering of human memories. Cereb. Cortex 21, 2534-2541.

Schabus, M., Dang-Vu, T. T., Albouy, G., Balteau, E., Boly, M., Carrier, J., Darsaud, A., Degueldre, C., Desseilles, M., Gais, S., Phillips, C., Rauchs, G., Schnakers, C., Sterpenich, V., Vandewalle, G., Luxen, A., and Maquet, P. (2007). Hemodynamic cerebral correlates of sleep spindles during human non-rapid eye movement sleep. Proc. Natl. Acad. Sci. U.S.A. 104, 13164-13169.

Schabus, M., Gruber, G., Parapatics, S., Sauter, C., Klosch, G., Anderer, P., Klimesch, W., Saletu, B., and Zeitlhofer, J. (2004). Sleep spindles and their significance for declarative memory consolidation. Sleep 27, 1479-1485.
Schabus, M., Hoedlmoser, K., Pecherstorfer, T., Anderer, P., Gruber, G., Parapatics, S., Sauter, C., Kloesch, G., Klimesch, W., Saletu, B., and Zeitlhofer, J. (2008). Interindividual sleep spindle differences and their relation to learning-related enhancements. Brain Res. 1191, 127-135.

Sejnowski, T. J., and Destexhe, A. (2000). Why do we sleep? Brain Res. 886, 208-223.

Shimamura, A. P. (2011). Episodic retrieval and the cortical binding of relational activity. Cogn. Affect. Behav. Neurosci. 11, 277-291.

Siapas, A., and Wilson, M. (1998). Coordinated interactions between hippocampal ripples and cortical spindles during slow-wave sleep. Neuron 21, 1123-1128.

Sirota, A., Csicsvari, J., Buhl, D., and Buzsáki, G. (2003). Communication between neocortex and hippocampus during sleep in rodents. Proc. Natl. Acad. Sci. U.S.A. 100, 2065-2069.

Skaggs, W. E., and McNaughton, B. L. (1996). Replay of neuronal firing sequences in rat hippocampus during sleep following spatial experience. Science 271, 1870-1873.

Smith, C. T., Aubrey, J. B., and Peters, K. R. (2004). Different roles for REM and stage 2 sleep in motor learning: A proposed model. Psychol. Belg. 44, 81-104.

Squire, L. R., and Alvarez, P. (1995). Retrograde amnesia and memory consolidation: a neurobiological perspective. Curr. Opin. Neurobiol. 5, 169-177.

Squire, L. R., Stark, C. E. L., and Clark, R. E. (2004). The medial temporal lobe. Annu. Rev. Neurosci. 27, 279-306.

Steriade, M. (2001). The Intact and Sliced Brain. Cambridge, MA: MIT Press.

Stickgold, R., and Walker, M. P. (2005). Memory consolidation and reconsolidation: what is the role of sleep? Trends Neurosci. 28, 408-415.

Taconnat, L., Baudouin, A., Fay, S., Raz, N., Bouazzaoui, B., El-Hage, W., Isingrini, M., and Ergis, A. M. (2010). Episodic memory and organizational strategy in free recall in unipolar depression: The role of cognitive support and executive functions. J. Clin. Exp. Neuropsychol. 32, 719-727.

Takashima, A., Petersson, K. M., Rutters, F., Tendolkar, I., Jensen, O., Zwarts, M. J., Mcnaughton, B. L., and Fernández, G. (2006). Declarative memory consolidation in humans: a prospective functional magnetic resonance imaging study. Proc. Natl. Acad. Sci. U.S.A. 103, 756-761. 
Tamaki, M., Matsuoka, T., Nittono, H., and Hori, T. (2008). Fast sleep spindle (13-15 hz) activity correlates with sleep-dependent improvement in visuomotor performance. Sleep 31, 204-211.

Tononi, G., and Cirelli, C. (2003). Sleep and synaptic homeostasis: a hypothesis. Brain Res. Bull. 62, 143-150.

Treves, A., Skaggs, W. E., and Barnes, C. A. (1996). How much of the hippocampus can be explained by functional constraints? Hippocampus 6 , 666-674.

Tronson, N., and Taylor, J. (2007). Molecular mechanisms of memory reconsolidation. Nat. Rev. Neurosci. 8, 262-275.

van der Helm, E., Gujar, N., Nishida, M., and Walker, M. P. (2011). Sleep-dependent facilitation of episodic memory details. PLoS ONE 6, e27421. doi:10.1371/journal.pone.0027421

Van Der Werf, Y. D., Altena, E., Schoonheim, M. M., Sanz-Arigita, E. J., Vis, J. C., De Rijke, W., and Van Someren, E. J. W. (2009). Sleep benefits subsequent hippocampal functioning. Nat. Neurosci. 12, 122-123. van Wingen, G. A., Van Eijndhoven, P., Cremers, H. R., Tendolkar, I., Verkes, R. J., Buitelaar, J. K., and Fernandez, G. (2010). Neural state and trait bases of mood-incongruent memory formation and retrieval in firstepisode major depression. J. Psychiatr. Res. 44, 527-534.

Wagner, U., Fischer, S., and Born, J. (2002). Changes in emotional responses to aversive pictures across periods rich in slow-wave sleep versus rapid eye movement sleep. Psychosom. Med. 64, 627-634.

Wagner, U., Gais, S., and Born, J. (2001). Emotional memory formation is enhanced across sleep intervals with high amounts of rapid eye movement sleep. Learn. Mem. 8, 112-119.

Wagner, U., Hallschmid, M., Rasch, B., and Born, J. (2006). Brief sleep after learning keeps emotional memories alive for years. Biol. Psychiatry 60, 788-790.

Wagner, U., Kashyap, N., Diekelmann, S., and Born, J. (2007). The impact of post-learning sleep vs. wakefulness on recognition memory for faces with different facial expressions. Neurobiol. Learn. Mem. 87, 679-687.

Walker, M., Stickgold, R., Alsop, D., Gaab, N., and Schlaug, G. (2005). Sleep-dependent motor memory plasticity in the human brain. $\mathrm{Neu}$ roscience 133, 911-917.

Walker, M. P. (2009). The role of sleep in cognition and emotion. Ann. N. Y. Acad. Sci. 1156, 168-197.

Walker, M. P., and van der Helm, E. (2009). Overnight therapy? The role of sleep in emotional brain processing. Psychol. Bull. 135, 731-748.

Wamsley, E. J., Tucker, M., Payne, J. D., Benavides, J. A., and Stickgold, R. (2010). Dreaming of a learning task is associated with enhanced sleep-dependent memory consolidation. Curr. Biol. 20, 850-855.

Wang, A. Y., and Thomas, M. H. (2000) Looking for long-term mnemonic effects on serial recall: the legacy of Simonides. Am. J. Psychol. 113, 331-340.

Werth, E., Achermann, P., Dijk, D. J., and Borbely, A. A. (1997). Spindle frequency activity in the sleep EEG: individual differences and topographic distribution. Electroencephalogr. Clin. Neurophysiol. 103 535-542.

Wilhelm, I., Diekelmann, S., and Born, J. (2008). Sleep in children improves memory performance on declarative but not procedural tasks. Learn. Mem. 15, 373-377.

Wilhelm, I., Diekelmann, S., Molzow, I., Ayoub, A., Mölle, M., and Born, J. (2011). Sleep selectively enhances memory expected to be of future relevance. J. Neurosci. 31, 1563-1569.

Wilson, M. A., and McNaughton, B. L. (1994). Reactivation of hippocampal ensemble memories during sleep. Science 265, 676-679.

Wylie, G. R., Foxe, J. J., and Taylor, T. L. (2008). Forgetting as an active process: an FMRI investigation of item-method-directed forgetting. Cereb. Cortex 18, 670-682.

Yoo, S., Hu, P., Gujar, N., Jolesz, F., and Walker, M. (2007a). A deficit in the ability to form new human memories without sleep. Nat. Neurosci. 10 385-392.
Yoo, S.-S., Gujar, N., Hu, P., Jolesz, F. A., and Walker, M. P. (2007b). The human emotional brain without sleep-a prefrontal amygdala disconnect. Curr. Biol. 17, R877-878.

Zeitlhofer, J., Gruber, G., Anderer, P., Asenbaum, S., Schimicek, P., and Saletu, B. (1997). Topographic distribution of sleep spindles in young healthy subjects. J. Sleep Res. 6, 149-155.

Conflict of Interest Statement: The authors declare that the research was conducted in the absence of any commercial or financial relationships that could be construed as a potential conflict of interest.

Received: 13 December 2011; paper pending published: 17 January 2012; accepted: 28 March 2012; published online: 01 May 2012.

Citation: Saletin JM and Walker MP (2012) Nocturnal mnemonics: sleep and hippocampal memory processing. Front. Neur. 3:59. doi: 10.3389/fneur.2012.00059

This article was submitted to Frontiers in Sleep and Chronobiology, a specialty of Frontiers in Neurology.

Copyright (c) 2012 Saletin and Walker. This is an open-access article distributed under the terms of the Creative Commons Attribution Non Commercial License, which permits non-commercial use, distribution, and reproduction in other forums, provided the original authors and source are credited. 